\title{
Registration of Lung Tissue Between Fluoroscope and CT Images: Determination of Beam Gating Parameters in Radiotherapy
}

\author{
Sukmoon Chang ${ }^{1,2}$, Jinghao Zhou ${ }^{2}$, Qingshan Liu ${ }^{2}$, Dimitris N. Metaxas ${ }^{2}$, \\ Bruce G. Haffty ${ }^{3}$, Sung N. Kim ${ }^{3}$, Salma J. Jabbour ${ }^{3}$, and Ning j. Yue ${ }^{3}$ \\ ${ }^{1}$ Computer Science, Capital College, Penn State University, PA, USA \\ ${ }^{2}$ Center for CBIM, Rutgers, The State University of New Jersey, NJ, USA \\ \{sukmoon, qsliu,dnm\}@cs.rutgers.edu, jhzhou@eden.rutgers.edu \\ 3 Dept. of Radiation Oncology, UMDNJ-RWJ Medical School, NJ, USA \\ $\{$ hafftybg, jabbousk, kim18, yuenj\}@umdnj.edu
}

\begin{abstract}
Significant research has been conducted in radiation beam gating technology to manage target and organ motions in radiotherapy treatment of cancer patients. As more and more on-board imagers are installed onto linear accelerators, fluoroscopic imaging becomes readily available at the radiation treatment stage. Thus, beam gating parameters, such as beam-on timing and beam-on window can be potentially determined by employing image registration between treatment planning CT images and fluoroscopic images. We propose a new registration method on deformable soft tissue between fluoroscopic images and DRR (Digitally Reconstructed Radiograph) images from planning CT images using active shape models. We present very promising results of our method applied to 30 clinical datasets. These preliminary results show that the method is very robust for the registration of deformable soft tissue. The proposed method can be used to determine beam-on timing and treatment window for radiation beam gating technology, and can potentially greatly improve radiation treatment quality.
\end{abstract}

\section{Introduction}

Radiation therapy is an important treatment modality for various cancers. The goals of radiation therapy can be better achieved by delivering conformal radiation dose to target volume while sparing normal or critical structures as much as possible. Various radiation delivery technologies, such as the three dimensional conformal radiation therapy (3DCRT) and the intensity modulated radiation therapy (IMRT), have been adopted and used to serve the purpose. However, the efficacy of these technologies may be compromised because of organ motion during treatment. It has been well known that organs and tumors located in the thorax and upper abdomen may exhibit significant respiratory induced motions [12. These physiologically related motions might well compromise the efficacy of radiation treatment of the tumors since larger margins are needed to provide adequate coverage of the targets, which may subsequently lead to 
increased toxicity and prevent dose escalation 34. Many techniques have been proposed and utilized to address the negative impact of respiratory motion during radiation treatment 567 . Although the method proposed in this paper is potentially applicable to all these techniques, we will limit our discussion only to the beam gating technique for simplicity.

In radiation therapy, treatment planning is usually conducted based on simulation CT images before treatment. At the planning stage, target volume is delineated on the CT image and the plan is designed so that the target volume will be adequately covered with radiation beams. The principle of using beam gating technology is to establish a feedback mechanism between tumor movement and radiation beam-on control during treatment: if the tumor moves outside the designed target volume, the radiation beam is turned off; otherwise, the radiation beam remains on.

In the current beam gating technology, the beam-on timing and treatment window are determined by a target surrogate that is placed outside the patient's body. With this method, to correctly deliver doses to the target, not only is a reliable correlation between the motions of the surrogate and the tumor required, but also this correlation needs to be reproducible from the simulation planning stage to the treatment stage. These two prerequisites, however, are difficult to satisfy. Another alternative is to implant a few markers into the target volume and use the markers as tracers to control the beam gating. The drawback of this approach is that it involves a surgical procedure and it may complicate the radiation treatment process. Furthermore, if the implanted markers migrate away from their original locations in the plan, not only is the tumor missed, but also normal tissue will be unnecessarily damaged.

As more and more on-board imagers are installed onto linear accelerators, fluoroscopic imaging becomes readily available at the radiation treatment stage. Since fluoroscopic imaging is able to detect target or organ motion, in this paper, we propose an automatic and robust registration method for soft tissues registration between fluoroscopic images and planning CT images (e.g., DRR). Then, we can derive temporal and spatial information of target volumes at the treatment stage. This information, instead of the artificial surrogate or implanted markers, can be used to determine beam-on timing and treatment window.

\section{Method}

Suppose that $N$ fluoroscopic images are acquired during a period of time at the treatment stage. Let $I\left(\boldsymbol{r}^{T}\left(t_{i}\right)\right)$ and $I\left(\boldsymbol{r}^{O}\left(t_{i}\right)\right)$ denote the representations of the target and the organ, respectively, on a fluoroscopic image $I$ taken at time $t_{i}, 1 \leq i \leq N$. Also let $I\left(\boldsymbol{r}_{\mathrm{CT}}^{\boldsymbol{T}}\right)$ and $I\left(\boldsymbol{r}_{\mathrm{CT}}^{\boldsymbol{O}}\right)$ denote the representations of the target and the organ, respectively, projected onto the digitally reconstructed radiographs (DRR) from the treatment planning $\mathrm{CT}$ or in $3 \mathrm{D} \mathrm{CT}$ volume. An image registration algorithm will need to search through $I\left(\boldsymbol{r}^{T}\left(t_{i}\right)\right)$ and $I\left(\boldsymbol{r}^{O}\left(t_{i}\right)\right)$ and determine $t_{k}$ such that an entropy function

$$
H\left[I\left(\boldsymbol{r}^{T}\left(t_{k}\right)\right)-I\left(\boldsymbol{r}_{\mathrm{CT}}^{\boldsymbol{T}}\right), I\left(\boldsymbol{r}^{O}\left(t_{k}\right)\right)-I\left(\boldsymbol{r}_{\mathrm{CT}}^{\boldsymbol{O}}\right)\right]
$$


reaches at its minimum. This time $t_{k}$ can be considered as the time at which the target and organ move to the locations that match those on the planning CT images. Therefore, to accurately deliver the radiation dose, the radiation beam needs to be turned on at $t_{k}$. However, in reality, if the beam were turned on only at time $t_{k}$, the gated radiation treatment would be prolonged to an unacceptable level. A practical approach to avoid the unacceptably prolonged treatment is to allow a beam-on window, during which the movements of the target and the organ are within a pre-specified margin $\delta$. This beam-on window $\left[t_{k_{1}}, t_{k_{2}}\right]$ can be determined by minimizing the following two entropy functions:

$$
\begin{aligned}
& H\left[I\left(\boldsymbol{r}^{T}\left(t_{k_{1}}\right)\right)-I\left(\boldsymbol{r}_{\mathrm{CT}}^{\boldsymbol{T}}-\boldsymbol{\delta}\right), I\left(\boldsymbol{r}^{O}\left(t_{k_{1}}\right)\right)-I\left(\boldsymbol{r}_{\mathrm{CT}}^{\boldsymbol{O}}-\boldsymbol{\delta}\right)\right] \\
& H\left[I\left(\boldsymbol{r}^{T}\left(t_{k_{2}}\right)\right)-I\left(\boldsymbol{r}_{\mathrm{CT}}^{\boldsymbol{T}}+\boldsymbol{\delta}\right), I\left(\boldsymbol{r}^{O}\left(t_{k_{2}}\right)\right)-I\left(\boldsymbol{r}_{\mathrm{CT}}^{\boldsymbol{O}}+\boldsymbol{\delta}\right)\right]
\end{aligned}
$$

Although various registration algorithms between fluoroscopic images and CT images have been proposed, the registration of soft tissue remains a major challenge. For example, [8] discussed a method based on robust similarity measurement, while 9 proposed a method based on both robust similarity measurement and optimization technique. In [10], a 3D volume is reconstructed from fluoroscopic images and compared to 3D CT. Soft tissues are not considered in any of these methods. In [11, deformable soft tissues are considered in the evaluation of the accuracy of similarity measurements; however, the image organ was a phantom spine and only a region of interest defined by user was registered.

In this section, we describe an automatic and robust method for the registration of deformable organs in medical images taken at different times using different modalities. Specifically, we propose a method for the registration of the lungs in fluoroscopic images to those in DRR images from planning CT. The proposed method consists of two steps: (1) accurate delineation of lung areas from both fluoroscopic and DRR images and (2) registration of the delineated lung areas. For the accurate delineation of lung areas, we use the active shape model approach with significant improvements [12,13,14,15].

\subsection{Active Shape Models (ASM) and Their Limitations}

An active shape model (ASM) represents the features of a shape as the point distribution model (PDM) [12. Given a set of training images, the feature of interest in each image is manually labeled with $n$ landmark points and represented as a vector in $2 n$-dimensional space, i.e., $\boldsymbol{x}=\left\langle x_{0}, y_{0}, x_{1}, y_{1}, \cdots, x_{n-1}, y_{n-1}\right\rangle$. After aligning these vectors into a common coordinate system, a set of orthogonal bases $\boldsymbol{P}$ is computed with the principal component analysis. Then, each aligned shape can be reconstructed as $\boldsymbol{x}=\overline{\boldsymbol{x}}+\boldsymbol{P} \boldsymbol{b}$, where $\overline{\boldsymbol{x}}$ and $\boldsymbol{b}$ are the mean shape and the shape parameter vector, respectively. This equation also allows us to search for a new example of the shape in an unlabeled image by varying $\boldsymbol{b}$ appropriately, often based on low-level image features such as the gradients along normal directions to the boundary of an initial shape toward the strongest edge in the image [12. Although it has been used successfully in many applications, ASM has two important limitations for the delineation of lung areas from both 


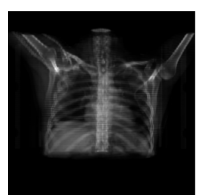

(a)

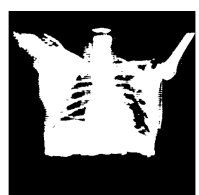

(b)

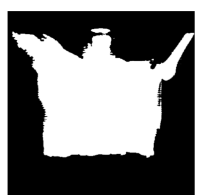

(c)

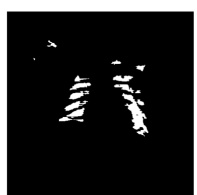

(d)

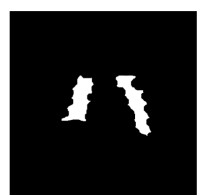

(e)

Fig. 1. The coarse segmentation of lung areas from DRR images. (a) Original DRR image, (b) Image after thresholding, (c) Image after morphological filling, (d) Image after XOR operation, and (e) Image after dilation and erosion operations.

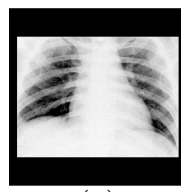

(a)

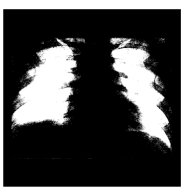

(b)

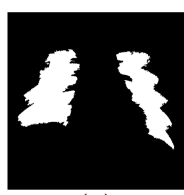

(c)

Fig. 2. The coarse segmentation of lung areas of fluoroscopic images. (a) Original fluoroscopic image, (b) Image after thresholding, (c) Image after dilation and erosion operations.

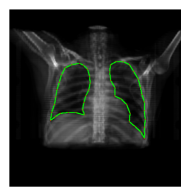

(a)

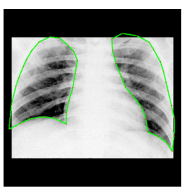

(b)

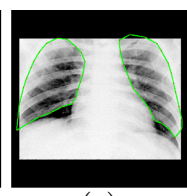

(c)
Fig. 3. Lung area segmentation. (a) and (b) DRR and fluoroscopic images by ASM with M-estimator, (c) Fluoroscopic image by ASM without M-estimator.

fluoroscopic and DDR images. In the next two sections, we will address these limitations and propose methods to overcome these drawbacks.

\subsection{Automatic Initialization of ASM}

The major drawback of ASM for searching for a new example of the shape in an unlabeled image is the initialization of the model. If the model is initialized too far from the feature of interest, the process may fail. To automate the accurate initialization of the model, we first rapidly extract the lung areas from unlabeled images by applying a series of morphological operators. The coarse segmentation process is illustrated in Figs. 1 and 2 ,

For DRR images (Fig. 1), the given image in (a) is first normalized and thresholded, using the mean intensity value of the normalized image as the threshold value, to generate a binary images shown in (b). Next, we perform the morphological filling on (b) to obtain (c). Then, we combine (b) and (c) with XOR operation to obtain (d). Finally, after applying dilation and erosion operations on (d), we obtain the coarse lung areas as shown in (e). For fluoroscopic images (Fig. 2), the given image in (a) is also normalized and thresholded, using the mean intensity value of the normalized image as the threshold value, to produce a binary image shown in (b). After applying dilation and erosion operations on (b), we obtain the coarse lung areas as shown in (c).

Note that our interest at this step is not to accurately segment the lung areas. The coarse lung areas obtained here are used only to automate the accurate initialization of the shape model on unlabeled images. We achieve the automatic 
and accurate initialization by aligning the centers of the shape models to the centers of the segmented coarse lung areas.

\subsection{Occluded Lung Area Segmentation}

Another limitation of ASM in finding an object in unlabeled images is that it heavily relies on the low-level image features to guide the search for the optimal positions of the feature points. For example, the gradient descent search on the image intensity profile has been widely used to move the model points toward the strongest edge in the image [12. However, this approach is not suitable for the accurate delineation of the lung areas in DDR and fluoroscopic images since the ribs occlude the lungs and appear as the strongest edge as can be seen in Fig 1(a) and 2(a). We overcome this difficulty by introducing a robust error function based on the M-estimator [13 16].

Given an orthogonal basis $\boldsymbol{P}$ obtained in Sec. 2.1. the projection $\boldsymbol{C}$ of a new example shape $\boldsymbol{X}$ is given by $\boldsymbol{C}=\boldsymbol{P}^{T} d \boldsymbol{X}$, where $\boldsymbol{X}=\overline{\boldsymbol{X}}+d \boldsymbol{X}$ and $\overline{\boldsymbol{X}}$ is the mean shape of the aligned shapes from the training images. Using the projection $\boldsymbol{C}$, we can also find a corresponding shape as $\hat{\boldsymbol{X}}=\overline{\boldsymbol{X}}+\boldsymbol{P} \boldsymbol{C}$, in which $\hat{\boldsymbol{X}}$ and $\boldsymbol{P C}$ approximates $\boldsymbol{X}$ and $d \boldsymbol{X}$, respectively. Therefore, in addition to optimizaing $\boldsymbol{X}$ and $d \boldsymbol{X}$ by the gradient descent search normal to the boundary only, our goal is to also find the optimal $\boldsymbol{C}$ by minimizing the robust energy function, $\boldsymbol{E}(\boldsymbol{C})=\min _{\boldsymbol{C}} \rho(\|d \boldsymbol{X}-\boldsymbol{P} \boldsymbol{C}\|, \sigma)$, where $\rho(x, \sigma)=x^{2} /\left(x^{2}+\sigma^{2}\right)$ is the Geman-McClure error function and $\sigma$ is a scale parameter that controls the convexity of the robust function. With an iterative gradient descent search on $\boldsymbol{E}$, we get $\boldsymbol{C}^{(n+1)}=\boldsymbol{C}^{(n)}+\lambda \Delta \boldsymbol{C}$, where $\lambda$ is a small constant that determines the step size and

$$
\Delta \boldsymbol{C}=\frac{\partial \boldsymbol{E}_{r p c a}}{\partial \boldsymbol{C}}=-2 \boldsymbol{P}(d \boldsymbol{X}-\boldsymbol{P} \boldsymbol{C}) \frac{\sigma^{2}}{\left(\|d \boldsymbol{X}-\boldsymbol{P C}\|^{2}\right)^{2}}
$$

By continuing the iterative process until $\left\|\boldsymbol{E}^{(n+1)}-\boldsymbol{E}^{(n)}\right\|<\epsilon$, where $\epsilon$ is a pre-selected tolerance, we obtain the optimal project $\boldsymbol{C}^{*}$ and a robust shape in the shape space as $\hat{\boldsymbol{X}}=\overline{\boldsymbol{X}}+\boldsymbol{P} \boldsymbol{C}^{*}$. Fig. 3 shows the typical results of the process applied to 30 clinical datasets, 25 of which were used as a training set. In the figure, (a) and (b) show the results of the ASM with M-estimator, where the lung areas occluded by the ribs are accurately segmented, while (c) shows the inaccruate result of the ASM without M-estimator.

\subsection{Registration of the Segmented Lung Areas}

Let $\boldsymbol{x}^{F}$ and $\boldsymbol{x}^{D}$ be the shape models of the lungs in fluoroscopic and DRR images obtained from the previous section, each containing $n$ landmark points. The registration of $\boldsymbol{x}^{F}$ onto $\boldsymbol{x}^{D}$ is achieved by finding the parameters of a $2 \mathrm{D}$ transformation that minimizes the weighted least square error:

$$
\epsilon=\sum_{i=0}^{n}\left(\boldsymbol{p}_{i}^{D}-\boldsymbol{M}\left(s, \theta, t_{x}, t_{y}\right) \cdot \boldsymbol{p}_{i}^{F}\right)^{T} \boldsymbol{W}\left(\boldsymbol{p}_{i}^{D}-\boldsymbol{M}\left(s, \theta, t_{x}, t_{y}\right) \cdot \boldsymbol{p}_{i}^{F}\right)
$$



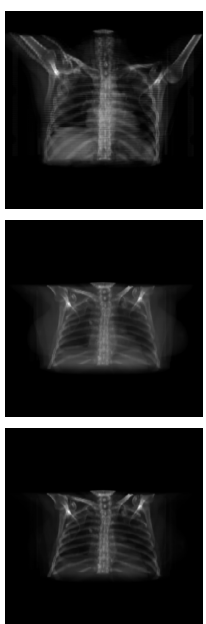

(a)
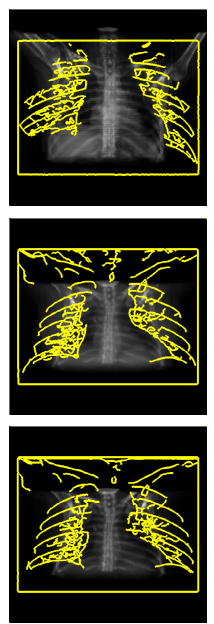

(b)
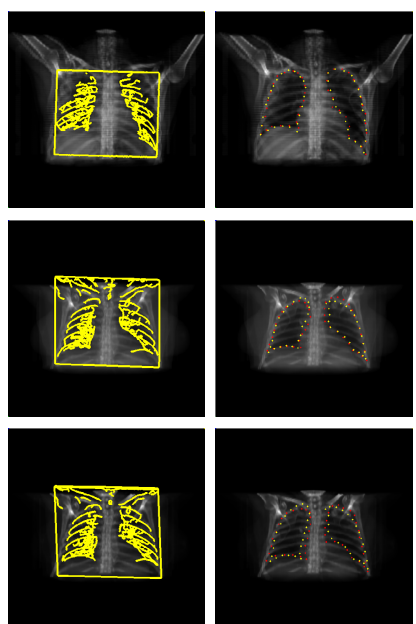

(c)
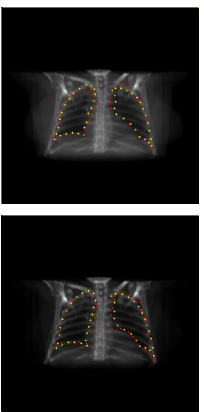

(d)
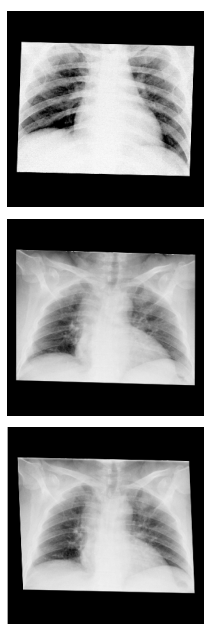

(e)

Fig. 4. Registration. (a) DRR image, (b) and (c) Fluoroscopic image before and after registration, superimposed on DRR image (only salient edges of the fluoroscopic image are shown for display purposes), (d) ASM contours on DRR image (red: ASM of DRR; yellow: ASM of registered fluoroscopic image), and (e) Registered fluoroscopic image.

where, $\boldsymbol{p}_{i}$ is the $i$-th point of a shape model in homogeneous coordinate system,

$$
M\left(s, \theta, t_{x}, t_{y}\right)=\left(\begin{array}{ccc}
s \cos \theta & -s \sin \theta & t_{x} \\
s \sin \theta & s \cos \theta & t_{y} \\
0 & 0 & 1
\end{array}\right)
$$

and $\boldsymbol{W}$ is a diagonal matrix of weights for each $\boldsymbol{p}_{i}$ that can be chosen to give more weights to the stable points [12. The results of the registration process are shown in Fig. 4 and discussed in the next section.

\section{Results}

We applied our method to 30 clinical datasets from different patients. Fig. 4 illustrates the results of the proposed method applied to three typical datasets. The given fixed images (DRR images) are shown in Fig.4(a). Fig. 4(b) shows the original fluoroscopic images superimposed on the corresponding fixed images before registration, while (c) shows the same fluoroscopic images after registration. Note that, in Fig. 4(b) and (c), only the salient edges of the fluoroscopic images are shown for display purposes. As can be seen from Fig. 4 (d), the ASM models from the fluoroscopic images are accurately registered onto the corresponding DRR images. Finally, Fig. 4(e) shows the fluoroscopic images transformed and registered to the corresponding fixed images.

The results are also summarized in Table1. The table shows that, on average, the mean distance and the root-mean-square error (RMSE) of the corresponding 
model points between the fixed image and the registered moving image are less than 7 pixels. The table also shows the overlap ratios of the lung areas between the DRR images and the registered fluoroscopic images. These overlap ratios can be used to determine the beam-on windows $\left[t_{k_{1}}, t_{k_{2}}\right]$ that minimize the two entropy functions in Eq. (2) and (3) given in Section 2 . For example, in the table, one of the fluoroscopic images registered onto the corresponding DRR image shows that the lung areas in the two images overlap well over $93 \%$. Thus, the time that this particular fluoroscopic image was taken can be interpreted as the $t_{k}$ that minimizes the entropy function in Eq. (1).

\section{Discussion}

We proposed an automatic and robust method for the registration of fluoroscopic images and DRR images from planning CT on deformable soft tissue, using an improved active shape model. We also presented various results of our method applied to 30 clinical datasets. With these promising results, the method can be used to determine beam-on timing and treatment window for beam gating technology without requiring a monitor or implanted markers. Since each of the fluoroscopic images is time stamped in segquence, the time that corresponds to the fluoroscopic image that matches the CT image set with the largest overlap ratio is when the radiation beam should be turned on. In this paper, we limited our discussion only to the beam gating techniques for simplicity. However, the proposed method is also applicable to the real-time target tracking as well as the dynamic multileaf collimator tracking.

Though the method is presented for the registration between fluoroscopic images and a 3D CT image set, the principle can also be applied to 4D computed tomography (4DCT) images. 4DCT technology has been introduced and becomes commercially available for clinical applications 1718. 4DCT images are usually reconstructed through time-resolved 3D CT data acquisition and contain spatiotemporal patient anatomic information. With the 4DCT technology, it is possible to obtain more information on organ and tumor motions at the simulation and planning stage. Theoretically, a dynamic treatment plan can be designed based on the 4DCT images, but the dynamically designed treatment requires higher degree of motion verification at the treatment stage. With the registration between the fluoroscopic images and 4DCT images, the derived

Table 1. Summary on 30 clinical datasets (MD, RMSE: mean distance and root mean square error of the corresponding points between two models; Overlap $(\mathrm{L} / \mathrm{R})$ : overlap ratios between two registered left (or, right) lung areas)

\begin{tabular}{|c||r|r|r|r|}
\hline Dataset & MD & RMSE & Overlap(L) & Overlap(R) \\
\hline \hline Best & 5.618 & 6.665 & 0.930 & 0.980 \\
\hline Worst & 9.515 & 10.163 & 0.819 & 0.821 \\
\hline Average & 6.036 & 6.979 & 0.880 & 0.901 \\
\hline
\end{tabular}


temporal and spatial information can be used not only for the determination of the gating parameters but also for the verification of motion pattern to ensure a safe dynamic treatment delivery of the highest possible quality.

\section{References}

1. Seppenwoolde, Y., et al.: Precise and real-time measurement of 3D tumor motion in lung due to breathing and heartbeat, measured during radiotherapy. Int. J. Padiat. Oncol. Biol. Phys. 53(4), 822-834 (2002)

2. Brandner, E., et al.: Abdominal organ motion measured using $4 \mathrm{D}$ CT. Int. J. Padiat. Oncol. Biol. Phys. 65(2), 554-560 (2006)

3. Bos, L., et al.: The sensitivity of dose distributions for organ motion and set-up uncertainties in prostate IMRT. Radiother. Oncol. 76, 18-26 (2005)

4. Hong, T., et al.: The impact of daily setup variations on head-and-neck intensitymodulated radiation therapy. Int. J. Radiat. Oncol. Biol. Phys. 61, 779-788 (2005)

5. Ozhasoglu, C., et al.: Issues in respiratory motion compensation during externalbeam radiotherapy. Int. J. Padiat. Oncol. Biol. Phys. 52, 1389-1399 (2002)

6. Wijesooriya, K., et al.: Determination of maximum leaf velocity and acceleration of a dynamic multileaf collimator: Implications for 4D radiotherapy. Med. Phys. 32, 932-941 (2005)

7. Vedam, S., Keall, P., Kini, V., Mohan, R.: Determining parameters for respiration gated radiotherapy. Med. Phys. 28, 2139-2146 (2001)

8. Livyatan, H., Yaniv, Z., Joskowicz, L.: Gradient-based 2-d/3-d rigid registration of fluoroscopic X-ray to CT. IEEE Trans. Med. Imag. 22(11), 1395-1406 (2003)

9. Zollei, L., et al.: 2d-3d rigid registration of X-ray fluoroscopy and CT images using mutual information and sparsely sampled histogram estimators. In: CVPR, pp. 696-703 (2001)

10. Tomazevic, D., Likar, B., Permus, F.: 3-d/2-d registration by integrating 2-d information in 3-d. IEEE Trans. Med. Imag. 25(1), 17-27 (2006)

11. Penney, G., et al.: A comparison of similarity measures for use in 2-d/3-d medical image registration. IEEE Trans. Med. Imag. 17(4), 586-595 (1998)

12. Cootes, T., Taylor, C., Cooper, D., Graham, J.: Active shape models - their training and application. Comp. Vis. Imag. Under. 61(1), 38-59 (1995)

13. Rogers, M., Graham, J.: Robust active shape model search. In: Heyden, A., Sparr, G., Nielsen, M., Johansen, P. (eds.) ECCV 2002. LNCS, vol. 2353, pp. 517-530. Springer, Heidelberg (2002)

14. Beichel, R., Bischof, H., Leberl, F., Sonka, M.: Robust active appearance models and their application to medical image analysis. IEEE Trans. Med. Imag. 24(9), 1151-1169 (2005)

15. van Ginneken, B., Stegmann, M., Loog, M.: Segmentation of anatomical structures in chest radiographs using supervised methods: A comparative study on a public database. Med. Imag. Anal. 10(1), 19-40 (2006)

16. De La Torre, F., Black, M.: A framework for robust subspace learning. Int. J. Comput. Vis. 54(1-3), 117-142 (2003)

17. Keall, P., et al.: Acquiring $4 \mathrm{D}$ thoracic CT scans using a multislice helical method. Phys. Med. Biol. 49, 2053-2067 (2004)

18. Rietzel, E., Pan, T., Chen, G.: 4D computed tomography: Image formation and clinical protocol. Med. Phys. 32, 874-889 (2005) 